\title{
Prevalence and distribution of human papillomavirus genotypes among women attending gynecology clinics in northern Henan Province of China
}

Xiangpeng Wang ${ }^{1}$, Yuan Song ${ }^{1}$, Xiaofei Wei ${ }^{1}$, Guanyu Wang ${ }^{1,2}$, Ruili Sun ${ }^{1 *}$, Mingyong Wang $^{1^{*}}$ and Lijun Zhao ${ }^{1 *}$ (D)

\begin{abstract}
Background: Human papillomavirus (HPV) infection can cause cervical and other cancers, including vulva, vagina, penis, anus, or oropharynx. However, in China's northern Henan Province, data on the prevalence and genotype distribution of HPV among women attending gynecology clinics is limited. This study aimed to investigate the current prevalence and genotype distribution of HPV among women attending gynecology clinics in northern Henan Province.
\end{abstract}

Methods: This study included 15,616 women aged 16-81 years old who visited the Xinxiang central hospital's gynecology department between January 2018 and December 2019. HPV DNA was detected by a conventional PCR method followed by HPV type-specific hybridization, which was designed to detect 17 high-risk HPV (HR-HPV) genotypes and 20 low-risk HPV (LR-HPV) genotypes. HPV prevalence and corresponding 95\% confidence intervals (95\% Cl) were calculated using SPSS 18.0.

Results: The overall HPV prevalence was 19.7\% among women in northern Henan Province. Single, double, and multiple HPV infections accounted for 13.7\%, 4.3\%, and 1.8\% of the total cases. Most infections were caused by HRHPV (71.8\%), and single genotype HPV infection (13.7\%) was the most common pattern. The most common HR-HPV genotype was HPV16 (4.3\%), followed by HPV52 (3.5\%) and HPV58 (2.0\%). The most common LR-HPV genotype was HPV6 (1.4\%), followed by HPV61 (1.1\%) and HPV81 (1.1\%).

Conclusions: HPV infection is high among women attending gynecology clinics in northern Henan Province. The highest prevalence was found in women less than 20 years old. In northern Henan Province, the 9-valent HPV vaccine is strongly recommended for regular immunization.

Keywords: Human papillomavirus, Genotype, Cervical cancer, Vaccine

*Correspondence: sunruili2016@126.com; wmy118@126.com; zhaolj2019@126.com

${ }^{1}$ Henan Key Laboratory of Immunology and Targeted Drugs, School of Laboratory Medicine, Xinxiang Medical University, Xinxiang 453003, Henan, China

Full list of author information is available at the end of the article

\section{Introduction}

Cervical cancer, ranked after breast cancer, colorectal cancer, and lung cancer, is the fourth most common cancer among women worldwide, with approximately 530,000 new cases and 275,000 deaths every year [1-3]. According to current data, nearly $85 \%$ of women's deaths from cervical cancer occurred in developing or underdeveloped countries [1]. In China, cervical cancer is ranked original author(s) and the source, provide a link to the Creative Commons licence, and indicate if changes were made. The images or other third party material in this article are included in the article's Creative Commons licence, unless indicated otherwise in a credit line to the material. If material is not included in the article's Creative Commons licence and your intended use is not permitted by statutory regulation or exceeds the permitted use, you will need to obtain permission directly from the copyright holder. To view a copy of this licence, visit http://creativecommons.org/licenses/by/4.0/. The Creative Commons Public Domain Dedication waiver (http://creativeco mmons.org/publicdomain/zero/1.0/) applies to the data made available in this article, unless otherwise stated in a credit line to the data. 
as the eighth most common killer for women and continues to be a public health problem affecting women's health [4]. Human papillomavirus (HPV) is a sexually transmitted virus, which is mainly passed on through genital contact and also passed on by skin-to-skin contact. HPV infection can cause cervical cancer and other cancers, including the vulva, vagina, penis, anus, or oropharynx. Persistent infection with HR-HPV is the major cause of cervical cancer. Globally, HPV infection has been involved in more than 99\% of cervical cancer [5]. HPV is a non-enveloped, double-stranded DNA virus with a genome of approximately $8.0 \mathrm{~kb}$. As of 9 March 2015, more than 200 different types of HPV, identified numerically, have been identified by the International HPV Reference Center [6]. Based on epidemiological and biological data, twelve HPV types $(16,18,31,33,35,39$, $45,51,52,56,58$ and 59) have been classified as HR-HPV [7]. Eight HPV types (26, 53, 66, 67, 68, 70, 73 and 82), identified as single HPV infection in about $3 \%$ cervical cancer, are classified as probable/possible (p) HR-HPV due to lack of biological data [8]. It is extremely difficult to isolate and culture HPV in vitro. Additionally, not all patients infected with HPV have an obvious antibody response. Thus, HPV DNA detection by PCR becomes a non-invasive and sensitive method for confirming cervical HPV infection. Since persistent infection with HR-HPV is a necessary cause for cervical cancer development, DNA detection and genotyping of HPV can be an essential method for controlling and preventing HPVrelated diseases in China.

Up to present, three licensed prophylactic vaccines, a bivalent vaccine against HPV16 and 18, a quadrivalent vaccine against HPV 6, 11, 16, and 18, and a 9-valent vaccine against HPV 6, 11, 16, 18, 31, 33, 45, 52 and 58, are considered as effective and safe for preventing HPV infection. However, the current vaccines offer protection only targeted at a few HPV genotypes and provide crossprotection for certain HPV genotypes. For example, the bivalent vaccine against HPV16 and 18 has a cross-protection against HPV 31, 34 and 45 [9]. The vaccines cannot prevent infection of all subtypes of HPV. Since the prevalence of HPV infection in women shows geographical distribution $[10,11]$, understanding HPV prevalence would be necessary to introduce an HPV vaccination program for cervical cancer prevention.

At present, the three vaccines against HPV can be used in China. However, only the bivalent vaccine and the quadrivalent vaccine against HPV are available in northern Henan Province. The 9-valent vaccines are only used in some provinces in China since May 2018. Lack of epidemiological data on HPV infection and genotype distribution makes it difficult to implement preventative measures such as HPV vaccination in northern Henan
Province. The sponsors and local government may support HPV vaccines by using the scientific data of HPV prevalence. Therefore, the prevalence and genotype distribution of HPV among women attending gynecology clinics in Henan Province were identified in this study. The results of this research are of great importance for estimation of the awareness of HPV infection and the introduction of vaccination program in northern Henan Province of China.

\section{Materials and methods \\ Study population and specimen collection}

Women aged 16 and 81 attending regular gynecological outpatient clinics in Xinxiang central hospital between January 2018 and December 2019 in northern Henan Province were invited to participate in this study. They were divided into six age groups. The G1 group meant age $<20$; the G2 group meant $20 \leq$ age $<30$; the G3 group meant $30 \leq$ age $<40$; the G4 group meant $40 \leq$ age $<50$; the G5 group meant $50 \leq$ age $<60$; the G6 group meant age $\geq 60$. Women were excluded for: the presence of cervical cancer, pregnancy at the time of enrollment, previous HPV vaccination, without age information, hysterectomy, or immunosuppression. Finally, a total of 15,616 participants were included in this study for analysis.

Cervical specimens were collected by the trained clinicians assisted by the speculum in Xinxiang central hospital. The endocervical and ectocervical cells were collected from the cervical canal using a plastic brush (Hybribio limited Corp, Chaozhou, Guangdong, China). The brush was placed into a $2 \mathrm{~mL}$ vial of Hybribio cervical cells preservation solution (Hybribio limited Corp, Chaozhou, Guangdong, China) for HPV DNA detection. This study was performed strictly following the Declaration of Helsinki and approved by the Ethics Committee in Xinxiang Medical University. Informed consent was obtained from all participants before enrollment.

\section{DNA extraction, PCR amplification, and HPV genotyping}

HPV DNA was extracted from the cervical cells using the Hybribio viral DNA extraction kit (Hybribio limited Corp, Chaozhou, Guangdong, China). Briefly, the cervical cells were first digested by proteinase $\mathrm{K}$. Then, the released DNA was obtained through absorption to magnetic glass particles, washed, and purified from these particles using the automated nucleic acid extraction instrument (Hybribio limited Corp, Chaozhou, Guangdong, China). The concentration and purity (OD260/ OD280 1.6-1.8) of DNA were determined by Nanodrop 2000 (Thermo Fisher Scientific, CA, USA). After the concentration was determined, the samples were stored. 
HPV DNA amplification and genotyping were conducted using a commercial Hybribio HPV genotyping detection kit (Hybribio limited Corp, Chaozhou, Guangdong, China) for 37 HPV types, including 17 HR-HPV types $(16,18,26,31,33,35,39,45,51,52,53$, $56,58,59,66,68,82)$ and 20 LR-HPV types $(6,11,34$, $40,42,43,44,54,55,57,61,67,69,70,71,72,73,81$, 83, 84). The China Food and Drug Administration has authorized the kit for clinical use. The HPV L1 consensus biotinylated primer sets were used in the PCR assay. $1 \mu \mathrm{L}$ of the DNA was used in the $25 \mu \mathrm{L}$ PCR master mix. PCR reaction was initiated with denaturation at $95^{\circ} \mathrm{C}$ for $9 \mathrm{~min}$, followed by 40 cycles of denaturation at $95^{\circ} \mathrm{C}$ for $20 \mathrm{~s}$, annealing at $55^{\circ} \mathrm{C}$ for $30 \mathrm{~s}$ and elongation at $72{ }^{\circ} \mathrm{C}$ for $30 \mathrm{~s}$, with a final extension at $72{ }^{\circ} \mathrm{C}$ for 5 min.

HPV genotyping was performed by the flow-through hybridization method. The flow-through hybridization was performed on a medical nucleic acid hybridization instrument prewarmed at $45{ }^{\circ} \mathrm{C}$ prior to usage. A nylon membrane on which $37 \mathrm{HPV}$ genotype-specific oligonucleotide probes secured was placed into the instrument. The biotinylated PCR product was denatured at $95^{\circ} \mathrm{C}$ for $5 \mathrm{~min}$ and then chilled on ice for $2 \mathrm{~min}$ before hybridization. The PCR product was mixed with the hybridization solutions, and the mixture was added into the sample wells to conduct flow-through hybridization for $10 \mathrm{~min}$. The nylon membrane was washed with the hybridization solution three times, and the empty region was blocked without reaction. The hybridizing signal was detected with streptavidin alkaline phosphatase, binding to biotinylated PCR products, and its subtract NBT/BCIP (nitro-blue tetrazolium-5-Bromo-4-chloro-3-indolyl phosphate). The genotyping result was detected by the position of the HPV-genotype probes on the membrane. The blue dot on the membrane, indicating a positive result, was judged by the naked eyes. The experimental procedures, including PCR and flow-through hybridization, followed the manufacturer's manual. Multiple dots showed multiple infections. Quality controls were carried out throughout the experiment, including PCR amplification and hybridization by using positive and negative controls provided by the kit.

\section{Statistical analysis}

HPV prevalence and genotype distribution were analyzed. Single, double, and multiple HPV infections were defined as infections with one, two, and more than two genotypes of HPV infections. HPV prevalence in designated groups and corresponding 95\% confidence intervals (95\% CI) were calculated using SPSS 18.0 for Windows (SPSS Inc., IL, USA).

\section{Results}

Overall and age-specific HPV prevalence

From January 2018 to December 2019, 15,616 cervical specimens collected for HPV DNA detection were used for statistical analysis. There were 3081 specimens positive for any HPV DNA, and the overall prevalence of HPV was 19.7\% (95\% CI 19.1-20.4\%). The 15,616 participants were divided into six age groups, and the HPV infection rate in each group was calculated as shown in Table 1. HPV infection was distributed in each age group with the infection rates from 17.7 to $41.8 \%$. The highest prevalence of HPV infection was found among women in the G1 group with an infection rate of $41.8 \%$ (95\% CI $36.5-47.2 \%$ ), followed by an infection rate of $22.9 \%$ (95\% CI $20.2-25.5 \%$ ) in the G6 group and an infection rate of $21.1 \%$ (95\% CI 19.6-25.5\%) in the G2 group.

Distribution of single, double, and multiple HPV infections Single genotype HPV infection (13.7\%) was the most common pattern, and it occurred more frequently than double (4.3\%) and multiple HPV infections (1.8\%) shown in Table 2. The group with the highest infection rate was the G1 group (28.0\%, 95\% CI 36.5-47.2\%). The infection rates declined as the age increased in the G6 group. Double infections had a high infection rate in G1 group at $10.8 \%$ and were also increasing with sharp decrease in G3 group. For multiple infections, the highest infection rate was in the G1 group (3.1\%) and the lowest in the G4 group (1.3\%).

\section{HPV genotype distribution}

There were 36 different HPV genotypes, including 17 HR-HPV genotypes and 19 LR-HPV genotypes, identified in this study. The prevalence of $17 \mathrm{HR}-\mathrm{HPV}$ was demonstrated in Table 3. The most common HR-HPV identified was HPV16 (4.3\%), followed by HPV52 (3.5\%), HPV58 (2.0\%), HPV53 (1.8\%) and HPV39 (1.5\%). To be noted, HPV18 was only the seventh most common HRHPV genotype to be detected. The top five genotypes for

Table 1 The prevalence of HPV among women in different groups

\begin{tabular}{lrccl}
\hline Group & Age, $\mathbf{y}$ & Sample size & Positive no & \% $(\mathbf{9 5} \% \mathbf{C l})$ \\
\hline G1 & $<20$ & 325 & 136 & $41.8(36.5-47.2)$ \\
G2 & $20-29$ & 2874 & 605 & $21.1(19.6-22.5)$ \\
G3 & $30-39$ & 4222 & 765 & $18.1(17.0-19.3)$ \\
G4 & $40-49$ & 4829 & 856 & $17.7(16.6-18.8)$ \\
G5 & $50-59$ & 2417 & 502 & $20.8(19.2-22.4)$ \\
G6 & $>60$ & 949 & 217 & $22.9(20.2-25.5)$ \\
Total & & 15,616 & 3081 & $19.7(19.1-20.4)$ \\
\hline
\end{tabular}


Table 2 Single, double, and multiple HPV infections in different groups

\begin{tabular}{|c|c|c|c|c|c|c|c|c|}
\hline \multirow[t]{2}{*}{ Group } & \multirow[t]{2}{*}{ Age, $y$} & \multirow[t]{2}{*}{ Sample size } & \multicolumn{2}{|c|}{ Single infection } & \multicolumn{2}{|c|}{ Double infections } & \multicolumn{2}{|c|}{ Multiple infections } \\
\hline & & & Positive no & $\%(95 \% \mathrm{Cl})$ & Positive no & $\%(95 \% \mathrm{Cl})$ & Positive no & $\%(95 \% \mathrm{Cl})$ \\
\hline G1 & $<20$ & 325 & 91 & $28.0(23.1-32.9)$ & 35 & $10.8(7.4-14.2)$ & 10 & $3.1(1.2-5.0)$ \\
\hline G2 & $20-29$ & 2874 & 396 & $13.8(12.5-15.0)$ & 151 & $5.3(4.4-6.1)$ & 58 & $2.0(1.5-2.5)$ \\
\hline G3 & 30-39 & 4222 & 562 & $13.3(12.3-14.3)$ & 142 & $3.4(2.8-3.9)$ & 61 & $1.4(1.1-1.8)$ \\
\hline G4 & $40-49$ & 4829 & 611 & $12.7(11.7-13.6)$ & 181 & $3.7(3.2-4.3)$ & 64 & $1.3(1.0-1.6)$ \\
\hline G5 & $50-59$ & 2417 & 331 & $13.7(12.3-15.1)$ & 115 & $4.8(3.9-5.6)$ & 56 & $2.3(1.7-2.9)$ \\
\hline G6 & $>60$ & 949 & 143 & $15.1(12.8-17.3)$ & 43 & $4.5(3.2-5.9)$ & 31 & $3.3(2.1-4.4)$ \\
\hline Total & & 15,616 & 2134 & $13.7(13.1-14.2)$ & 667 & $4.3(4.0-4.6)$ & 280 & $1.8(1.6-2.0)$ \\
\hline
\end{tabular}

Table 3 Distribution of HR-HPV genotypes in study participants

\begin{tabular}{|c|c|c|c|c|c|c|c|}
\hline \multirow[t]{2}{*}{ HPV type } & \multicolumn{2}{|c|}{ Single infection } & \multicolumn{2}{|c|}{ Double infections } & \multicolumn{2}{|c|}{ Multiple infections } & \multirow{2}{*}{$\begin{array}{l}\text { Total infections } \\
\%(95 \% \mathrm{Cl})\end{array}$} \\
\hline & Positive no & $\%(95 \% \mathrm{Cl})$ & Positive no & $\%(95 \% \mathrm{Cl})$ & Positive no & $\%(95 \% \mathrm{Cl})$ & \\
\hline HPV16 & 380 & $2.4(2.2-2.7)$ & 184 & $1.2(1.0-1.3)$ & 105 & $0.7(0.5-0.8)$ & $4.3(4.0-4.6)$ \\
\hline HPV52 & 185 & $1.2(1.0-1.4)$ & 98 & $0.6(0.5-0.8)$ & 269 & $1.7(1.5-1.9)$ & $3.5(3.2-3.8)$ \\
\hline HPV58 & 144 & $0.9(0.8-1.1)$ & 106 & $0.7(0.5-0.8)$ & 61 & $0.4(0.3-0.5)$ & $2.0(1.8-2.2)$ \\
\hline HPV53 & 138 & $0.9(0.7-1.0)$ & 81 & $0.5(0.4-0.6)$ & 59 & $0.4(0.3-0.5)$ & $1.8(1.6-2.0)$ \\
\hline HPV39 & 95 & $0.6(0.5-0.7)$ & 90 & $0.6(0.5-0.7)$ & 45 & $0.3(0.2-0.4)$ & $1.5(1.3-1.7)$ \\
\hline HPV51 & 88 & $0.6(0.4-0.7)$ & 69 & $0.4(0.3-0.5)$ & 61 & $0.4(0.3-0.5)$ & $1.4(1.2-1.6)$ \\
\hline HPV18 & 96 & $0.6(0.5-0.7)$ & 32 & $0.2(0.1-0.3)$ & 40 & $0.3(0.2-0.3)$ & $1.1(0.9-1.2)$ \\
\hline HPV33 & 74 & $0.5(0.4-0.6)$ & 53 & $0.3(0.2-0.4)$ & 32 & $0.2(0.1-0.3)$ & $1.0(0.9-1.2)$ \\
\hline HPV31 & 70 & $0.4(0.3-0.6)$ & 46 & $0.3(0.2-0.4)$ & 29 & $0.2(0.1-0.3)$ & $0.9(0.8-1.1)$ \\
\hline HPV68 & 57 & $0.4(0.3-0.5)$ & 38 & $0.2(0.2-0.3)$ & 27 & $0.2(0.1-0.2)$ & $0.8(0.6-0.9)$ \\
\hline HPV66 & 55 & $0.4(0.3-0.4)$ & 31 & $0.2(0.1-0.3)$ & 33 & $0.2(0.1-0.3)$ & $0.8(0.6-0.9)$ \\
\hline HPV56 & 43 & $0.3(0.2-0.4)$ & 33 & $0.2(0.1-0.3)$ & 35 & $0.2(0.1-0.3)$ & $0.7(0.6-0.8)$ \\
\hline HPV59 & 27 & $0.2(0.1-0.2)$ & 20 & $0.1(0.1-0.2)$ & 23 & $0.1(0.1-0.2)$ & $0.4(0.3-0.6)$ \\
\hline HPV45 & 38 & $0.2(0.2-0.3)$ & 10 & $0.1(0.0-0.1)$ & 10 & $0.1(0.0-0.1)$ & $0.4(0.3-0.5)$ \\
\hline HPV35 & 18 & $0.1(0.1-0.2)$ & 10 & $0.1(0.0-0.1)$ & 12 & $0.1(0.0-0.1)$ & $0.3(0.2-0.3)$ \\
\hline HPV82 & 14 & $0.1(0.0-0.1)$ & 15 & $0.1(0.0-0.1)$ & 8 & $0.1(0.0-0.1)$ & $0.2(0.2-0.3)$ \\
\hline HPV26 & 15 & $0.1(0.0-0.1)$ & 13 & $0.1(0.0-0.1)$ & 5 & $0.0(0.0-0.1)$ & $0.2(0.1-0.3)$ \\
\hline
\end{tabular}

individuals with a single HR-HPV infection were HPV16, HPV52, HPV58, HPV53, and HPV39. For individuals with double HPV infections, the HR-HPV that ranked top five were HPV16, HPV58, HPV52, HPV39, and HPV53. For individuals with multiple HPV infections, the HRHPV that ranked top five were HPV52, HPV16, HPV58, HPV51, and HPV53. These data suggested that HPV16 infection was predominant in HPV-positive patients. The prevalence of 19 LR-HPV genotypes was demonstrated in Table 4. The most common LR-HPV identified was HPV6, followed by HPV61, HPV81, HPV54, and HPV11. The most commonly detected genotype for individuals with a single LR-HPV infection was HPV61, followed by HPV54, HPV6, HPV81, and HPV11. For double and multiple HPV-infected individuals, HPV6 and HPV61 were the two most common LR-HPV. The prevalent genotypes ranked top 10 were HPV16, HPV52, HPV58, HPV53, HPV39, HPV51, HPV6, HPV18, HPV61, and HPV81. HR-HPV infection accounted for $71.8 \%$, demonstrating the most infection was caused by HR-HPV in northern Henan Province.

\section{Discussion}

China has a vast territory and a large population of more than 1.4 billion, and the prevalence of HPV varies greatly in different provinces of China. Some studies reported the prevalence and genotype distribution of HPV in different provinces in China [12-15]. However, there are few reports on HPV prevalence in Henan Province. The present study provided the prevalence and genotype data 
Table 4 Distribution of LR-HPV genotypes in study participants

\begin{tabular}{|c|c|c|c|c|c|c|c|}
\hline \multirow[t]{2}{*}{ HPV type } & \multicolumn{2}{|c|}{ Single infection } & \multicolumn{2}{|c|}{ Double infections } & \multicolumn{2}{|c|}{ Multiple infections } & \multirow{2}{*}{$\begin{array}{l}\text { Total infections } \\
\%(95 \% \mathrm{Cl})\end{array}$} \\
\hline & Positive no & $\%(95 \% \mathrm{Cl})$ & Positive no & $\%(95 \% \mathrm{Cl})$ & Positive no & $\%(95 \% \mathrm{Cl})$ & \\
\hline HPV6 & 78 & $0.5(0.4-0.6)$ & 88 & $0.6(0.4-0.7)$ & 52 & $0.3(0.2-0.4)$ & $1.4(1.2-1.6)$ \\
\hline HPV61 & 111 & $0.7(0.6-0.8)$ & 27 & $0.2(0.1-0.2)$ & 30 & $0.2(0.1-0.3)$ & $1.1(0.9-1.2)$ \\
\hline HPV81 & 70 & $0.4(0.3-0.6)$ & 55 & $0.4(0.3-0.4)$ & 43 & $0.3(0.2-0.4)$ & $1.1(0.9-1.2)$ \\
\hline HPV54 & 79 & $0.5(0.4-0.6)$ & 51 & $0.3(0.2-0.4)$ & 32 & $0.2(0.1-0.3)$ & $1.0(0.9-1.2)$ \\
\hline HPV11 & 49 & $0.3(0.2-0.4)$ & 35 & $0.2(0.1-0.3)$ & 32 & $0.2(0.1-0.3)$ & $0.7(0.6-0.9)$ \\
\hline HPV40 & 48 & $0.3(0.2-0.4)$ & 23 & $0.1(0.1-0.2)$ & 9 & $0.1(0.0-0.1)$ & $0.5(0.4-0.6)$ \\
\hline HPV84 & 27 & $0.2(0.1-0.2)$ & 26 & $0.2(0.1-0.2)$ & 25 & $0.2(0.1-0.2)$ & $0.5(0.4-0.6)$ \\
\hline HPV34 & 27 & $0.2(0.1-0.2)$ & 14 & $0.1(0.0-0.1)$ & 15 & $0.1(0.0-0.1)$ & $0.4(0.3-0.5)$ \\
\hline HPV70 & 16 & $0.1(0.1-0.2)$ & 16 & $0.1(0.1-0.2)$ & 10 & $0.1(0.0-0.1)$ & $0.3(0.2-0.4)$ \\
\hline HPV44 & 20 & $0.1(0.1-0.2)$ & 12 & $0.1(0.0-0.1)$ & 9 & $0.1(0.0-0.1)$ & $0.3(0.2-0.3)$ \\
\hline HPV42 & 21 & $0.1(0.1-0.2)$ & 9 & $0.1(0.0-0.1)$ & 8 & $0.1(0.0-0.1)$ & $0.2(0.2-0.3)$ \\
\hline HPV55 & 10 & $0.1(0.0-0.1)$ & 16 & $0.1(0.1-0.2)$ & 3 & $0.0(0.0-0.0)$ & $0.2(0.1-0.3)$ \\
\hline HPV73 & 12 & $0.1(0.0-0.1)$ & 5 & $0.0(0.0-0.1)$ & 11 & $0.1(0.0-0.1)$ & $0.2(0.1-0.2)$ \\
\hline HPV43 & 4 & $0.0(0.0-0.1)$ & 8 & $0.1(0.0-0.1)$ & 9 & $0.1(0.0-0.1)$ & $0.1(0.1-0.2)$ \\
\hline HPV69 & 8 & $0.1(0.0-0.1)$ & 6 & $0.0(0.0-0.1)$ & 4 & $0.0(0.0-0.1)$ & $0.1(0.1-0.2)$ \\
\hline HPV57 & 4 & $0.0(0.0-0.1)$ & 9 & $0.1(0.0-0.1)$ & 0 & $0.0(0.0-0.0)$ & $0.1(0.0-0.1)$ \\
\hline HPV83 & 5 & $0.0(0.0-0.1)$ & 3 & $0.0(0.0-0.0)$ & 3 & $0.0(0.0-0.0)$ & $0.1(0.0-0.1)$ \\
\hline HPV67 & 3 & $0.0(0.0-0.0)$ & 1 & $0.0(0.0-0.0)$ & 4 & $0.0(0.0-0.1)$ & $0.1(0.0-0.1)$ \\
\hline HPV71 & 5 & $0.0(0.0-0.1)$ & 1 & $0.0(0.0-0.0)$ & 2 & $0.0(0.0-0.0)$ & $0.1(0.0-0.1)$ \\
\hline
\end{tabular}

of HPV from women attending regular gynecological outpatient clinics from January 2018 to December 2019 in the northern Henan Province of China. Our study showed that the overall HPV infection rate was $19.7 \%$, which was similar to the results from several surveys of HPV prevalence reported in other provinces of China [16-18]. The prevalence of HPV in northern Henan Province was lower than that reported in Shandong Province (28.4\%) and Fujian Province (38.3\%) [19, 20], but higher than Yunnan Province (12.9\%) and Shanxi Province $(8.92 \%)[12,21]$. The different HPV prevalence in northern Henan Province was expected and could have been due to the different economic conditions, living habits and customs, cultural diversity, sampling strategy, and the HPV detection methods [13, 22]. According to the previous reports, the HPV infection rate ranges from 6.7 to $44.5 \%$ in China [23]. Although the HPV infection rates in northern Henan Province are not the highest in China, the women need education on prevention and control of HPV infection and the related diseases.

This present study provided age-specific HPV infection rates among women. Our results showed that women under 20 years had the highest infection rate. Single, double and multiple infections rates were also the highest in women under 20 years. There are two possible reasons for this phenomenon: the first reason is that literature shows that HPV prevalence is higher in the younger age group [22, 24]. The second reason is that younger women may engage in unprotected sexual activities, exposing them to infections. The above two reasons led to the highest infection rate in the G1 group. It was reported that young women infected with HPV were temporary, and the immune system would clear the virus in most cases $[25,26]$. Therefore, the infection rate of HPV will gradually decline with age increased.

The present study found that the prevalence of HPV declined gradually in the middle-aged groups and slightly increased in the G6 group (22.9\%), which indicated that women over 60 years suffered severely from HPV infection. For women over 60 years, HPV infections may occur when they are young, and the infections probably are persistent infections instead of new HPV infections. If the HPV cannot be eliminated by the immune system and persist for several years, this will present as a risk factor for neoplasia development. HPV infection rates was $21.1 \%$ in women aged $20-29,18.1 \%$ in women aged $30-39,17.7 \%$ in women aged $40-49$ and $20.8 \%$ in women aged 50-59. According to recent data from China National Cancer Center, there were 98,990 new cases of cervical cancer and 30,500 deaths in China in 2015 [27]. The number of new cervical cancer cases was 10,948 in Henan Province, with an incidence of 21.09 per 100,000 [28]. Since HPV infection is a major etiological factor for cervical cancer development, future treatment that could 
eliminate HPV infection and prevent the progression of cervical cancer is of great importance.

Our present study also provided the age-specific distribution of single, double, and multiple HPV infections rates. Except for the G1 group, the single, double, and multiple HPV infections rates of the rest groups were very close. There has not been a consensus on whether numerous HPV infections raise the risk of cervical cancer more than a single HPV infection up until now. Some studies have reported that multiple HPV infections had a higher risk of cervical cancer occurrence and development than single HPV infections [29, 30]. However, other studies have found that single HPV infections had a greater risk of developing cervical cancer concerning multiple infections $[31,32]$. In our study, the prevalence of single HPV infections is higher than that of double and multiple HPV infections, accounting for $13.7 \%$ of the total cases, and HPV16 was the most commonly detected genotype in a single HPV infection. HPV16 infection is the one with the highest oncogenic risk [32]. Thus, more attention should be paid to single HPV16 infection. The double HPV infections and multiple HPV infections accounted for $4.3 \%$ and $1.8 \%$ of the total cases, respectively. The investigation of double and multiple HPV infections is of great importance to study the prevalence of HPV and is also of great significance to develop a multivalent $\mathrm{HPV}$ vaccine.

It is important to know the HPV genotypes distribution since data concerning the distribution of HPV genotypes is concerning with the vaccine development. Persistent infection with HR-HPV is the primary etiological factor for cervical cancer. Up to $70 \%$ of cervical cancers are caused by HPV16 and HPV18, with the remainder of cervical cancers caused by other HR-HPV genotypes. In the present study, HPV16, HPV52, HPV58, HPV53, and HPV39 were the top five HR-HPV genotypes. HPV16 ranked first in our study, and the infection rate reached up to $4.3 \%$. HPV52 and HPV58 ranked second and third, respectively. Besides HPV16, both HPV52 and HPV58 are also strongly correlated with cervical cancer development [31]. The top three genotypes distribution pattern is consistent with the data from several previous Chinese population-based HPV investigations [19, 33, 34]. It was reported the HPV18 ranked second in Chinese women between 1991 and 2016 [4]. In our study, the HPV18, with an infection rate of $1.1 \%$, ranked seven in the HRHPV genotypes, which was consistent with the recent results in several other regions in China [19, 21, 22].

Furthermore, the study also investigated the distribution of LR-HPV. Our study showed that the top five most common LR-HPV genotypes were HPV6, HPV61, HPV81, HPV54, and HPV11. For the LR-HPV types, cervical cancer association is very rare in the general women population. LR-HPV mainly causes genital warts, common warts, flats warts, and many other skin lesions [35], which are usually asymptomatic but are sometimes accompanied by inching, burning, or bleeding, leading to psychosocial disturbances. The 9-valent HPV vaccine is used to prevent infection with HPV6, HPV11, HPV16, HPV18, HPV31, HPV33, HPV45, HPV52, and HPV58. Our results support the recommendation of the 9-valent HPV vaccine for routine vaccination in northern Henan Province. Meanwhile, vaccines against HPV53, HPV39, and HPV51 should also be developed for women in this area.

\section{Conclusions}

A high prevalence of HPV was reported in the study. HPV16, HPV52, and HPV58 were the dominant HRHPV in northern Henan Province. The results in our study provide important information for cervical cancer screening and vaccination in women in northern Henan Province of China.

\section{Abbreviations}

HPV: Human papillomavirus; HR-HPV: High-risk HPV; LR-HPV: Low-risk HPV; 95\% Cl: 95\% Confidence intervals.

\section{Acknowledgements}

We thank the individuals who volunteered to participate in the research. We are grateful to the staff from Xinxiang central hospital in Xinxiang city for their generous help. We express our gratitude to those who contributed to the article's publication.

\section{Authors' contributions}

XPW, RLS, MYW, and LJZ designed and supervised the research. XPW, YS, XFW, and GYW acquisition of data. RLS, MYW, and LJZ analyzed and interpreted the data. XPW prepared the manuscript. All authors read and approved the final manuscript.

\section{Funding}

This study was supported by the Natural Science Foundation of China (No. 81802837), the Program for Ph.D. Starting Research Funding from Xinxiang Medical University (No. XYBSKYZZ 201616), the Program for Innovative

Research Team (in Science and Technology) in University of Henan Province (No. 20IRTSTHN030), Natural Science Foundation of Henan Province for Distinguished Young Scholars (No. 212300410013) and the Key Scientific Research Projects of Higher Education Institutions in Henan Province (No. 19A320005).

\section{Availability of data and materials}

The data collected from Xinxiang central hospital in Xinxiang city can be freely shared. Any additional information may be obtained from the corresponding author on a reasonable request.

\section{Declarations}

Ethics approval and consent to participate

This study was approved by the Ethics Committee in Xinxiang Medical University. Informed consent was obtained from all participants before enrollment.

Consent for publication

Not applicable. This manuscript does not contain any person's data in any form (including individual details, images, or videos. 


\section{Competing interests}

The authors declare that they have no competing interests.

\section{Author details}

${ }^{1}$ Henan Key Laboratory of Immunology and Targeted Drugs, School of Laboratory Medicine, Xinxiang Medical University, Xinxiang 453003, Henan, China. ${ }^{2}$ Department of Clinical Laboratory, Xinxiang Central Hospital, Xinxiang 453000, Henan, China.

Received: 10 September 2021 Accepted: 13 December 2021

Published online: 06 January 2022

\section{References}

1. Small W Jr, Bacon MA, Bajaj A, Chuang LT, Fisher BJ, Harkenrider MM, Jhingran A, Kitchener HC, Mileshkin LR, Viswanathan AN, Gaffney DK. Cervical cancer: a global health crisis. Cancer. 2017;123(13):2404-12.

2. Arbyn M, Weiderpass E, Bruni L, de Sanjosé S, Saraiya M, Ferlay J, Bray F. Estimates of incidence and mortality of cervical cancer in 2018: a worldwide analysis. Lancet Glob Health. 2020;8(2):e191-203.

3. Olusola P, Banerjee HN, Philley JV, Dasgupta S. Human papilloma virusassociated cervical cancer and health disparities. Cells. 2019;8(6):622.

4. Zhou HL, Zhang W, Zhang CJ, Wang SM, Duan YC, Wang JX, Yang H, Wang $X Y$. Prevalence and distribution of human papillomavirus genotypes in Chinese women between 1991 and 2016: a systematic review. J Infect. 2018;76(6):522-8

5. Wang $X$, Huang $X$, Zhang $Y$. Involvement of human papillomaviruses in cervical cancer. Front Microbiol. 2018;9:2896.

6. Kocian BJ, Bzhalava D, Forslund O, Dillner J, Poljak M. Molecular methods for identification and characterization of novel papillomaviruses. Clin Microbiol Infect. 2015;21(9):808-16.

7. Galati L, Brancaccio RN, Gupta P, Lohmann E, Robitaille A, Mandishora RSD, Cuenin C, Filotico R, Combes JD, Giuliano AR, Donà MG, Tommasino $M$, Gheit T. Diversity of human papillomavirus in the anal canal of HIVpositive and HIV-negative men. J Infect. 2021;82(1):112-6.

8. Halec G, Alemany L, Lloveras B, Schmitt M, Alejo M, Bosch FX, Tous S, Klaustermeier JE, Guimerà N, Grabe N, Lahrmann B, Gissmann L, Quint W, Bosch FX, de Sanjose S, Pawlita M. Pathogenic role of the eight probably/ possibly carcinogenic HPV types $26,53,66,67,68,70,73$ and 82 in cervical cancer. J Pathol. 2014;234(4):441-51.

9. Pollock KGJ. Sustained cross-protection of the bivalent human papillomavirus vaccine. J Infect Dis. 2018;217(10):1515-6.

10. Bao HL, Jin C, Wang S, Song Y, Xu ZY, Yan X, Li LM, Ning Y, Wang HJ. Prevalence of cervicovaginal human papillomavirus infection and genotypes in the pre-vaccine era in China: a nationwide population-based study. J Infect. 2021;84(2):75-83.

11. Bogale AL, Belay NB, Medhin G, Ali JH. Molecular epidemiology of human papillomavirus among HIV infected women in developing countries: systematic review and meta-analysis. Virol J. 2020;17(1):179.

12. Li Z, Liu F, Cheng S, Shi L, Yan Z, Yang J, Shi L, Yao Y, Ma Y. Prevalence of HPV infection among 28457 Chinese women in Yunnan Province, southwest China. Sci Rep. 2016;6:21039.

13. Luo L, He P, Liu Q, Jiang Y, Zhang Y, Li Q, Li Q, Li S, Yang F, Ling H, Dai X, Li $\mathrm{Z}$, Chen $\mathrm{H}$. Prevalence and genotype distribution of HPV infection among 214,715 women from Southern China, 2012-2018: baseline measures prior to mass HPV vaccination. BMC Infect Dis. 2021;21(1):328.

14. Li H, Li P, Huang L, Sun L, Ren H, Li P. Prevalence characteristics of cervical human papillomavirus (HPV) infection in Zhoupu District, Shanghai City, China. Virol J. 2020;17(1):84.

15. Zhang P, Liu S, Zhong Z, Hou J, Lin L, Weng R, Su L, Lei N, Hou T, Yang H. Prevalence and genotype distribution of human papillomavirus infection among women in northeastern Guangdong Province of China. BMC Infect Dis. 2018;18(1):204

16. Ge Y, Zhong S, Ren M, GeY, Mao Y, Cao P. Prevalence of human papillomavirus infection of 65,613 women in East China. BMC Public Health. 2019;19(1):178.

17. $\mathrm{Xu} \mathrm{HH}$, Lin A, Chen YH, Dong SS, Shi WW, Yu JZ, Yan WH. Prevalence characteristics of cervical human papillomavirus (HPV) genotypes in Taizhou area, China: a cross-sectional study of 37967 women from the general population. BMJ Open. 2017;7(6):e014135.
18. Mai Q, Yang X, Cheng H, Wu G, Wu Z. Prevalence and genotype distribution of human papillomavirus among women with cervical lesions in Shenzhen city. China Hum Vaccin Immunother. 2021;17(4):965-71.

19. Jiang L, Tian X, Peng D, Zhang L, Xie F, Bi C, Wang R, Wang J, Qi D. HPV prevalence and genotype distribution among women in Shandong Province, China: analysis of 94489 HPV genotyping results from Shandong's largest independent pathology laboratory. PLoS ONE. 2019;14(1):e0210311.

20. Wu C, Zhu X, Kang Y, Cao Y, Lu P, Zhou W, Zhou H, Zhang Y, Song Y. Epidemiology of human papilloma virus infection among women in Fujian China. BMC Public Health. 2017;18(1):95.

21. Yang J, Wang W, Wang Z, Wang Z, Wang Y, Wang J, Zhao W, Li D, Liu H, Hao M. Prevalence, genotype distribution and risk factors of cervical HPV infection in Yangqu, China: a population-based survey of 10086 women. Hum Vaccin Immunother. 2020;16(7):1645-52.

22. Zhang C, Cheng W, Liu Q, Guang Q, Zhang Q. Distribution of human papillomavirus infection: a population-based study of cervical samples from Jiangsu Province. Virol J. 2019;16(1):67.

23. Li K, Li Q, Song L, Wang D, Yin R. The distribution and prevalence of human papillomavirus in women in mainland China. Cancer. 2019;125(7):1030-7.

24. Hao S, Wang C, Liu S, He J, Jiang Y. HPV genotypic spectrum in Jilin province, China, where non-vaccine-covered HPV53 and 51 are prevalent, exhibits a bimodal age-specific pattern. PLOS ONE. 2020;15(3):e0230640.

25. Garbuglia AR, Lapa D, Sias C, Capobianchi MR, Del Porto P. The use of both therapeutic and prophylactic vaccines in the therapy of papillomavirus disease. Front Immunol. 2020;11:188.

26. Ferrall $L$, Lin KY, Roden RBS, Hung CF, Wu TC. Cervical cancer immunotherapy: facts and hopes. Clin Cancer Res. 2021;27(18):4953-73.

27. Zhang S, Xu H, Zhang L, Qiao Y. Cervical cancer: epidemiology, risk factors and screening. Chin J Cancer Res. 2020;32(6):720-8.

28. He J, Xu H, Dai Y, Zheng L, Sun L, Guo L, Hu X, Sun X, Meng L, Chen Q, Zhang S. Incidence and mortality of cervical cancer in Henan Province, 2016. J Cancer Control Treat. 2021;34(7):611-6.

29. Li Y, Wang H, Zhang Y, Jing X, Wu N, Hou Y, Hao C. Correlation between multi-type human papillomavirus infections and viral loads and the cervical pathological grade. Int J Gynaecol Obstet. 2021;152(1):96-102.

30. Adcock R, Cuzick J, Hunt WC, McDonald RM, Wheeler CM. Role of HPV genotype, multiple infections, and viral load on the risk of high grade cervical neoplasia. Cancer Epidemiol Biomark Prev. 2019;28(11):1816-24.

31. Li M, Du X, Lu M, Zhang W, Sun Z, Li L, Ye M, Fan W, Jiang S, Liu A, Wang M, Meng Y, Li Y. Prevalence characteristics of single and multiple HPV infections in women with cervical cancer and precancerous lesions in Beijing, China. J Med Virol. 2019;91(3):473-81.

32. Bruno MT, Scalia G, Cassaro N, Boemi S. Multiple HPV16 infection with two strains: a possible marker of neoplastic progression. BMC Cancer. 2020;20(1):444

33. Zhang J, Cheng K, Wang Z. Prevalence and distribution of human papillomavirus genotypes in cervical intraepithelial neoplasia in China: a metaanalysis. Arch Gynecol Obstet. 2020;302(6):1329-37.

34. Yuan XW, Li YJ, Qiu Q, Luo Z, Zhao X. Prevalence and genotype distribution of human papillomavirus among 9945 women from the Nanhai area of Foshan. BMC Infect Dis. 2019:19(1):71.

35. Egawa N, Doorbar J. The low-risk papillomaviruses. Virus Res. 2017:231:119-27.

\section{Publisher's Note}

Springer Nature remains neutral with regard to jurisdictional claims in published maps and institutional affiliations. 